\title{
How does the spatial structure of habitat loss affect the eco-epidemic dynamics?
}

\author{
Min Su ${ }^{a}$, Cang Hui ${ }^{b}$, Yanyu Zhang ${ }^{c}$, Zizhen $\mathrm{Li}^{a, c, *}$ \\ a School of Mathematics and Statistics, Lanzhou University, Lanzhou 730000, China \\ ${ }^{b}$ Center of Excellence for Invasion Biology, Department of Botany and Zoology, University of Stellenbosch, \\ Private Bag X1, Matieland 7602, South Africa \\ ${ }^{\mathrm{c}}$ Key Laboratory of Arid and Grassland Agroecology of the Ministry of Education, School of Life Sciences, \\ Lanzhou University, Lanzhou 730000, China
}

\section{A R T I C L E I N F O}

\section{Article history:}

Received 3 November 2007

Received in revised form

31 August 2008

Accepted 5 September 2008

Published on line 1 November 2008

\section{Keywords:}

Eco-epidemiology

Habitat loss

Spatial structure

Spatial correlation

Lattice model

Join-count statistics

\begin{abstract}
A B S T R A C T
Habitat loss is considered as one of the primary causes of species extinction, especially for a species that also suffers from an epidemic disease. Little attention has been paid to the combined effect of habitat loss and epidemic transmission on the species spatiotemporal dynamics. Here, a spatial model of the parasite-host/prey-predator eco-epidemiological system with habitat loss was studied. Habitat patches in the model, instead of undergoing a random loss, were spatially clustered by different degrees. Not only the quantity of habitat loss but also its clustering degree was shown to affect the equilibrium of the system. The infection rate and the probability of successful predation were keys to determine the spatial patterns of species. The epidemic disease is more likely to break out if only a small amount of suitable patches were lost. Counter-intuitively, infected preys are more sensitive to habitat loss than predators if the lost patches are highly clustered. This result is new to eco-epidemiology and implies a possibility of using spatial arrangement of suitable (or unsuitable) patches to control the spread of epidemics in the ecological system.
\end{abstract}

(c) 2008 Elsevier B.V. All rights reserved.

\section{Introduction}

Habitat loss, mainly caused by anthropogenic disturbances, has been considered one of the most devastating factors to threaten the persistence of species on this planet (Fahrig and Merriam, 1994; Bascompte and Solé, 1998; Swihart et al., 2001; Prakash and de Roos, 2002; Szwabiński and Pekalski, 2006). The effect of habitat loss on species persistence has been thoroughly studied (Noss and Murphy, 1995; Durrett and Levin, 1996; Melián and Bascompte, 2002; Lin, 2005). Because species are interlinked by various interactions in a community, habitat loss can cause a substantial chain reaction on species dynamics, making it important for closer examination.

Most previous studies of habitat loss have focused on the response of species in terms of their temporal dynamics (for single species, Bascompte and Solé, 1998; for multiple species, Tilman, 1994; Namba et al., 1999; Swihart et al., 2001; for food webs, Melián and Bascompte, 2002). A general conclusion from these studies is that there exists a correlation between species trophic rank and their extinction threshold; that is, the species in the highest trophic level or the superior in competition suffers the most from habitat loss (e.g., Holt et al., 1999; Melián and Bascompte, 2002; Nakagiri and Tainaka, 2004). A parallel

\footnotetext{
* Corresponding author at: School of Mathematics and Statistics, Lanzhou University, Lanzhou 730000, China. Tel.: +86 9318913370 ; fax: +869318912823 .

E-mail addresses: sum04@lzu.cn (M. Su), zizhenlee@lzu.edu.cn (Z. Li). 0304-3800/\$ - see front matter @ 2008 Elsevier B.V. All rights reserved.
} doi:10.1016/j.ecolmodel.2008.09.009 


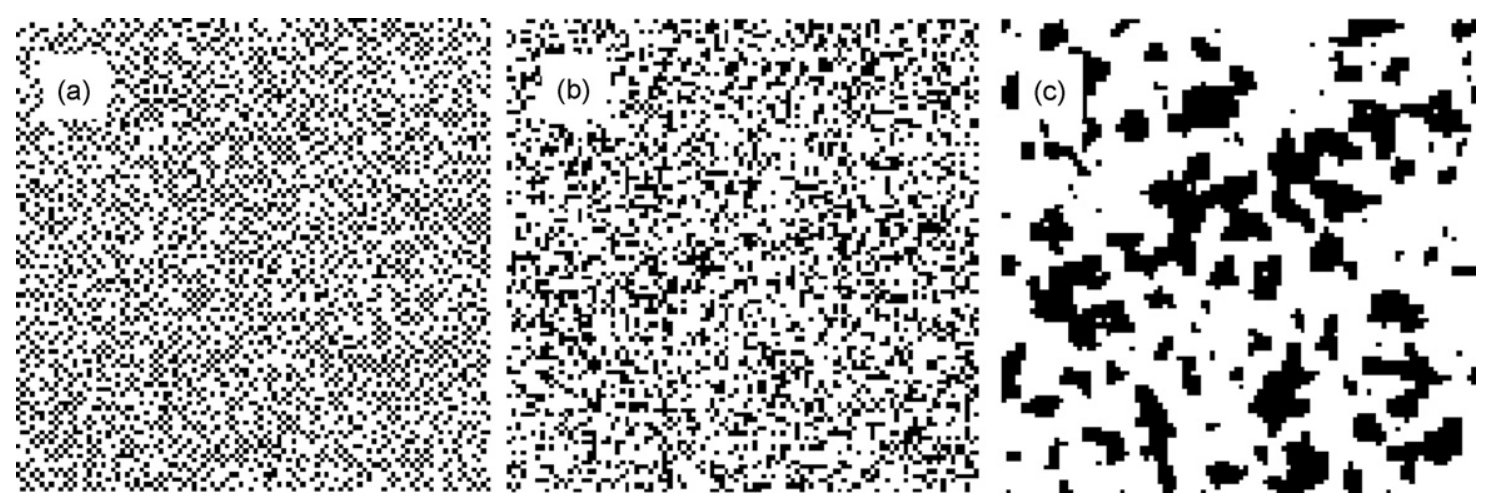

Fig. 1 - Three artificially generated landscapes in $100 \times 100$ lattices, each of which has $30 \%$ of habitat loss (black) and $70 \%$ of suitable patches (white). (a) A segregated distribution of lost patches $\left(q_{u / u}=0.05\right)$; (b) a random distribution $\left(q_{u} / \mathrm{u}=0.3\right) ;(c)$ an aggregated distribution $\left(q_{\mathrm{u} / \mathrm{u}}=0.8\right)$.

correlation has also been identified in behavior ecology that the habitat loss and certain spatial structures can be beneficial to altruistic individuals that are inferior to cheating individuals in an evolutionary game (Nowak et al., 1994; Zhang et al., 2005; Hui and McGeoch, 2007). In epidemiology, Murray (1993) also showed that the habitat loss can interfere with the epidemic transmission. In some cases, habitat loss can totally extinguish the epidemic disease (Garrett et al., 2007). However, few studies are available to analyze the effect of habitat loss on both an ecological and an epidemiological system, i.e. in the field of eco-epidemiology (Bairagi et al., 2007).

Habitat loss has normally been analyzed by making a stipulated fraction of patches unavailable for species (e.g., Namba et al., 1999; Prakash and de Roos, 2002; Szwabiński and Pekalski, 2006), whereas the lost patches are considered to be spatially random in the landscape. This contrasts the non-random structure of realistic habitat, displaying a certain degree of spatial correlation (or aggregation). Recent works have shown that an increase of the degree of spatial clustering of unsuitable patches can increase the population size (Hiebeler, 2000, 2004; Hiebeler and Morin, 2007). Given that the epidemic disease (or parasite) can also strongly regulate the host population (Chattopadhyay and Bairagi, 2001; Hethcote et al., 2004; Pal et al., 2006; Bairagi et al., 2007; Su et al., 2008a), we here demonstrate the effect of habitat loss on such a parasite-host/prey-predator eco-epidemiological system. Specifically, we address how the amount and the clustering degree of habitat loss affect the dynamics, spatial structure and the extinction threshold of the parasitic disease, host preys and predators. A spatially stochastic lattice model on a partially destroyed habitat with different clustering levels is designed accordingly, which considers species local interactions and dispersal, as well as the demographic stochasticity.

\section{Model}

To examine how the spatial structure of habitat loss affects the eco-epidemic dynamics, a parasite-host (prey)-predator model was constructed in a lattice landscape. Following
Hiebeler (2000) and Hiebeler and Morin (2007), we simulated this heterogeneous lattice landscape by defining two habitat states, i.e. suitable and unsuitable, in which the unsuitable patches cannot be occupied. The proportion of unsuitable patches in the landscape is described as the variable $p_{\mathrm{u}}$, which indicates the amount of habitat loss. The clustering parameter is given by $q_{u} / u$, representing the first-order spatial-autocorrelation of the lost (unsuitable) patches. This clustering parameter measures the probability that a randomly chosen neighbor of an unsuitable patch is also unsuitable (Satō and Iwasa, 2000; Hui et al., 2006). Therefore, the proportion of suitable patches is $p_{\mathrm{s}}=1-p_{\mathrm{u}}$, and the clustering degree of suitable patches is $q_{\mathrm{s} / \mathrm{s}}=1-p_{\mathrm{u}}\left(1-q_{\mathrm{u} / \mathrm{u}}\right) /\left(1-p_{\mathrm{u}}\right)$. A heterogeneous landscape with a certain amount of unsuitable patches can be fully described using $p_{\mathrm{u}}$ and $q_{\mathrm{u} / \mathrm{u}}$. For example, we presented in Fig. 1 three typical landscapes using Hiebeler's (2000) algorithm, with all having $p_{u}=0.3$ but different clustering degrees $q_{u} / u$. The local dynamics and interactions of a parasite-host (prey)-predator system were then incorporated into a patch-occupant lattice (individual-based) model on this heterogeneous landscape. The synchronous updating and von Neumann (four nearest patches) neighborhood were adopted (Hui and Li, 2003; Hui and McGeoch, 2007). Periodic boundaries were used to exclude the edge effect (e.g., Sayama, 2004; Hui and Li, 2004).

Considering a parasitic disease that infects the prey/host population, we thus have five possible states for each patch $p_{t}(i, j)$ : unsuitable $(=0)$, suitable but empty $(=1)$, occupied by a susceptible prey $(=2)$, occupied by an infected prey $(=3)$, or occupied by a predator $(=4)$. Detailed rules governing the dynamics of this eco-epidemiological model are as follows:

(1) Initially, the populations (of susceptible preys, infected preys, and predators) are randomly located in the suitable patches (note that the spatial structure at the equilibrium of the system is insensitive to the initial condition; Hiebeler and Morin, 2007);

(2) Only susceptible prey can colonize the empty suitable patches at a probability of $r$ in each time step. Specifically, if the chosen patch is empty and suitable, it becomes occupied by the offspring reproduced from the neighboring 
susceptible prey at a probability of $1-(1-r)^{N_{S}}$ (Rhodes and Anderson, 1997; Tilman and Kareiva, 1997), where $N_{S}$ $(=0,1,2,3,4)$ is the total number of susceptible prey neighbors. Notably, the colonization probability of a patch with two susceptible prey neighbors, $2 r-r^{2}$, is less than double the probability of a patch with one susceptible prey neighbor, $2 r$, indicating a density-dependent phenomenon.

(3) The infection of parasitic disease among prey can be described by a Susceptible-Infection (SI) model (Packer et al., 2003; Deredec and Courchamp, 2006; Bairagi et al., 2007). The susceptible preys can be infected at a probability of $q$ through the contact between the susceptible and infected individuals. As a result, a susceptible prey can become infected at a probability of $1-(1-q)^{N_{I}}$, where $N_{\text {I }}(=0,1,2,3,4)$ is the sum of patches occupied by infected prey in the neighborhood.

(4) The predator not only consumes the susceptible prey (at a probability of $p$ ), but also consumes the infected one (at a probability of $w p$ ). It is further assumed that predators are prudent will avoid the prey with infected disease, $0<w<1$ (Packer et al., 2003; Bairagi et al., 2007). Accordingly, a patch with a susceptible prey can be foraged by predators at a probability of $1-(1-p)^{N_{P}}$, and the probability is $1-(1-w p)^{N_{P}}$ for patches with infected prey, where $N_{P}(=0,1,2,3,4)$ is the total number of neighboring predators.

(5) The transition rules of the patch status from time $t$ to $t+1$ were given in Table 1.

To describe the spatial structure of the simulation results from the above model, we selected a simple spatialautocorrelation index, namely the join-count statistics (JCS; Fortin et al., 2002). Through counting the occupancy and spatial correlation (i.e. the clustering degree), we used JCS to classify the distributions as spatially aggregated, segregated or random (Satō and Iwasa, 2000; Hui et al., 2006), which are consistent with the definition of the spatial structure of the

Table 1 - Probabilities of state transition of patches in the lattice model.

\begin{tabular}{ll}
$p_{t}(i, j) \rightarrow p_{t+1}(i, j)$ & \multicolumn{1}{c}{ Probability } \\
\hline $1 \rightarrow 2$ & $1-(1-r)^{N_{S}}$ \\
$1 \rightarrow 1$ & $(1-r)^{N_{S}}$ \\
$2 \rightarrow 1$ & $d_{1}$ \\
$2 \rightarrow 3$ & $1-(1-q)^{N_{I}}$ \\
$2 \rightarrow 4$ & $1-(1-p)^{N_{P}}$ \\
$2 \rightarrow 2$ & $(1-q)^{N_{I}}+(1-p)^{N_{P}}-d_{1}-1$ \\
$3 \rightarrow 1$ & $d_{2}$ \\
$3 \rightarrow 4$ & $1-(1-w p)^{N_{P}}$ \\
$3 \rightarrow 3$ & $(1-w p)^{N_{P}}-d_{2}$ \\
$4 \rightarrow 1$ & $d_{3}$ \\
$4 \rightarrow 4$ & $1-d_{3}$ \\
\end{tabular}

Note: $r$ is the reproduction probability of a susceptible prey; $q$ represents the probability that an infected individual transmits disease to its susceptible neighbors; $p$ and $w p$ are the probabilities that a predator successfully hunts a susceptible neighboring prey or a infected prey; $d_{1}, d_{2}$ and $d_{3}$ are the extinction probability of the susceptible prey, the infected prey and the predator; $N_{S}, N_{I}$ and $N_{P}$ are the number of neighbors of the susceptible prey, the infected prey and the predator. landscape above. Occupancy $P_{\sigma}$ is the proportion of patches being occupied by population $\sigma$. The clustering degree $Q_{\sigma / \sigma^{\prime}}$ is the conditional probability that a randomly chosen nearest neighbor of a patch in state $\sigma^{\prime}$ is found in state $\sigma$ (Satō and Iwasa, 2000). The capital notation $P_{\sigma}$ and $Q_{\sigma / \sigma^{\prime}}$ will be used when discussing the population model to distinguish from $p_{u}$, $p_{\mathrm{s}}$ and $q_{\mathrm{u} / \mathrm{u}}, q_{\mathrm{s} / \mathrm{s}}$ for habitat types. Therefore, spatially explicit aggregation can be described by the JCS as $C_{\sigma}=Q_{\sigma / \sigma}-P_{\sigma}>0$, implying the positive first-order spatial correlation between two adjacent occupied patches. The spatial random distribution has $C_{\sigma}=0$ and indicates the independence of two adjacent, occupied patches. The spatial segregated distribution has $C_{\sigma}<0$ and indicates a negative spatial correlation between two adjacent patches (Hui et al., 2006; Hui and McGeoch, 2007).

To reveal the effect of habitat loss on the dynamics of an eco-epidemiological system, we introduced two separate perturbation experiments at the time $t=2000$. The first was to introduce a $20 \%$ random habitat loss $\left(p_{u}=0.2, q_{u} / \mathrm{u}=0.2\right)$ to the landscape. The second perturbation was to introduce the same habitat loss of $20 \%$ but with a much higher clustering degree $\left(p_{u}=0.2, q_{u} / u=0.75\right)$. At each perturbation, a new landscape was generated using Hiebeler's (2000) algorithm. We excluded the situation of a segregated habitat loss since no evidence in literature points to its possibility in reality.

Since the predation rate (the probability of $p$ per time step) and the infection rate (the probability of $q$ per time step) have been shown to play a vital role in the system behavior (Melián and Bascompte, 2002; Kondoh, 2003; Pal et al., 2006), we also examined the equilibrium of the eco-epidemiological system in three combinations of these two parameters under different degrees of habitat loss. Specifically, the amount of habitat loss, $p_{\mathrm{u}}$, varied from 0.05 to 0.9 with an interval of 0.05 ; whereas the clustering degree of habitat loss was fixed $\left(q_{\mathrm{u} / \mathrm{u}}=0.6\right)$.

We further examined the occupancy of the susceptible prey $\left(P_{S}\right)$, the occupancy of the infected prey $\left(P_{I}\right)$, the occupancy of the predator $\left(P_{P}\right)$ and the incidence of the parasitic disease in the host/prey populations $P_{\mathrm{I}} /\left(P_{\mathrm{S}}+P_{\mathrm{I}}\right)$, as well as the JCS of these three populations $\left(C_{S}, C_{I}\right.$ and $\left.C_{P}\right)$, as a function of the spatial structure of the landscape, $p_{\mathrm{u}}$ and $q_{\mathrm{u} / \mathrm{u}}$. To obtain the equilibrium of the system, the simulation was run for 4000 time steps for 324 combinations of $P_{u}(=0.05,0.1, \ldots, 0.9)$ and $q_{\mathrm{u} / \mathrm{u}}(=0.05,0.1, \ldots, 0.9)$ in a $100 \times 100$ two-dimensional lattice network. Eight replicates were run for each combination of $p_{\mathrm{u}}$ and $q_{\mathrm{u} / \mathrm{u}}$

To explore the vulnerability of different populations in response to the habitat loss, we analyzed the extinction threshold of three species. Extinction threshold can be defined as the minimum amount of habitat loss in which the species are doomed to extinct. When the proportion of the unsuitable habitat is less than the extinction threshold, the population can sustain itself. Specifically, we calculated the extinction threshold for the three populations under different clustering degree of habitat loss, $q_{\mathrm{u} / \mathrm{u}}$. The extinction threshold under various amounts of habitat loss $\left(p_{\mathrm{u}}\right)$ was not shown here since it has been described as the Levins rule in the metapopulation studies (Levins, 1969; Bascompte and Solé, 1998; Hui and Li, 2003). 

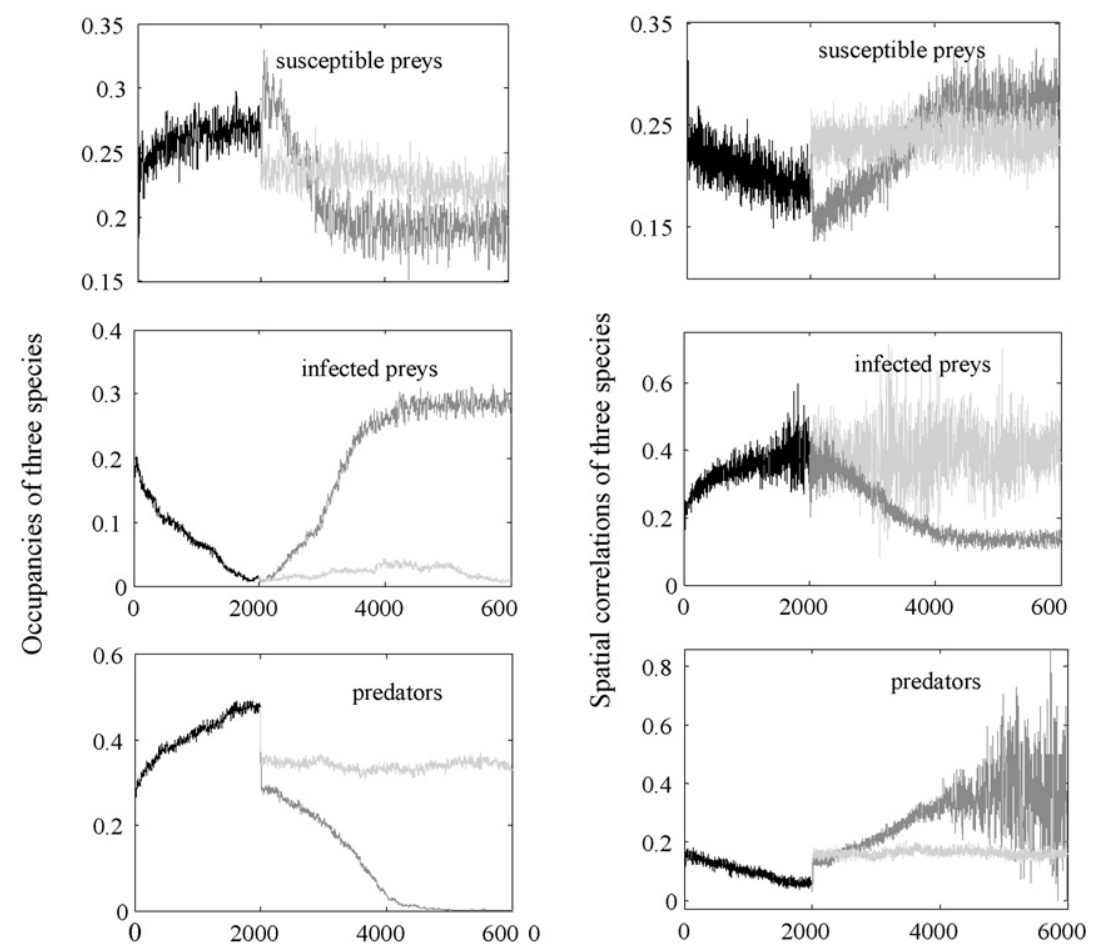

Fig. 2 - The population dynamics of the spatially eco-epidemiological model in $100 \times 100$ lattices. The temporal dynamics of occupancies and spatial correlations for populations are shown under two perturbation experiments. The landscape under the first perturbation changes from no habitat loss at time $t=2000$ to a fragmented landscape with randomly distributed lost patches $\left(p_{u}=0.2, q_{u} / \mathrm{u}=0.2\right)$, the second perturbation differs only in its high clustering degree $\left(q_{\mathrm{u} / \mathrm{u}}=0.75\right)$. Black lines represent no habitat loss, gray lines represent the random perturbation and light gray lines clustered perturbation. Parameter values are: $r=0.5, p=0.11, q=0.25, w=0.5, d_{1}=0.05, d_{2}=0.11, d_{3}=0.092$.

\section{Results}

In Fig. 2, the temporal dynamics of the occupancy and spatial correlation of susceptible preys, infected preys and predators were presented under the two perturbation experiments. After the random perturbation, the occupancy of susceptible preys increased initially, but later it dropped into a new stationary state. The initial increase was due to the sharp decrease in the occupancy of predators; whereas the subsequent decrease was because of the rapid transmission of the parasitic disease. Meanwhile, the occupancy of the infected prey increased dramatically, but that of the predator declined abruptly and became close to extinct eventually (the left panel of Fig. 2). After the clustered perturbation, both the occupancies of susceptible preys and the predators decrease dramatically, with the infected prey showing no signs of change (Fig. 2). Comparing the dynamics of occupancies after these two perturbations, we concluded that the equilibriums under these two perturbations are different. This indicates that the spatial structure (i.e. the clustering degree) of lost patches has a dramatic influence on the eco-epidemiological system (the left panel of Fig. 2).

The spatial correlation of populations also differed markedly under these two perturbations (the right panel of Fig. 2). After the random perturbation, the spatial correlation of the susceptible prey decreased initially, and then increased into a new equilibrium; whereas the spatial correlation of the infected prey declined dramatically (Fig. 2). The spatial correlation of predators also exhibited a strong oscillation due to the fairly low occupancy of predator populations. Results from the clustered perturbation showed that the spatial correlation of susceptible preys and predators increased and that of the infected prey showed a stronger oscillation than under the random perturbation. Therefore, the spatial structure of habitat loss also strongly affects the spatial structure of population distribution. The spatial structure of habitat loss can have profound effects on species dynamics (their equilibriums and oscillations) and distribution.

The equilibriums of the system under three different levels of the predation probability $(p)$ and the infection probability (q) were shown in Fig. 3. When the predation probability was high, the infected prey went extinct due to over-predation (comparing Fig. 3b with Fig. 3a). When the infection probability was high, the predators also went extinct due to lack of prey (comparing Fig. 3c with Fig. 3a). Furthermore, the two remaining populations in Fig. $3 b$ (the susceptible prey and predator) showed similar patterns to those in Fig. 3a, but the infected prey in Fig. 3c declined monotonically, contrast to Fig. 3a. These two alternative results can also be illustrated as an intraguild predation model formed by the parasite-host interaction and the prey-predator interaction (Fig. 4; see Section 4). Therefore, the consequences of habitat loss depend on the inner interactions of the system. 


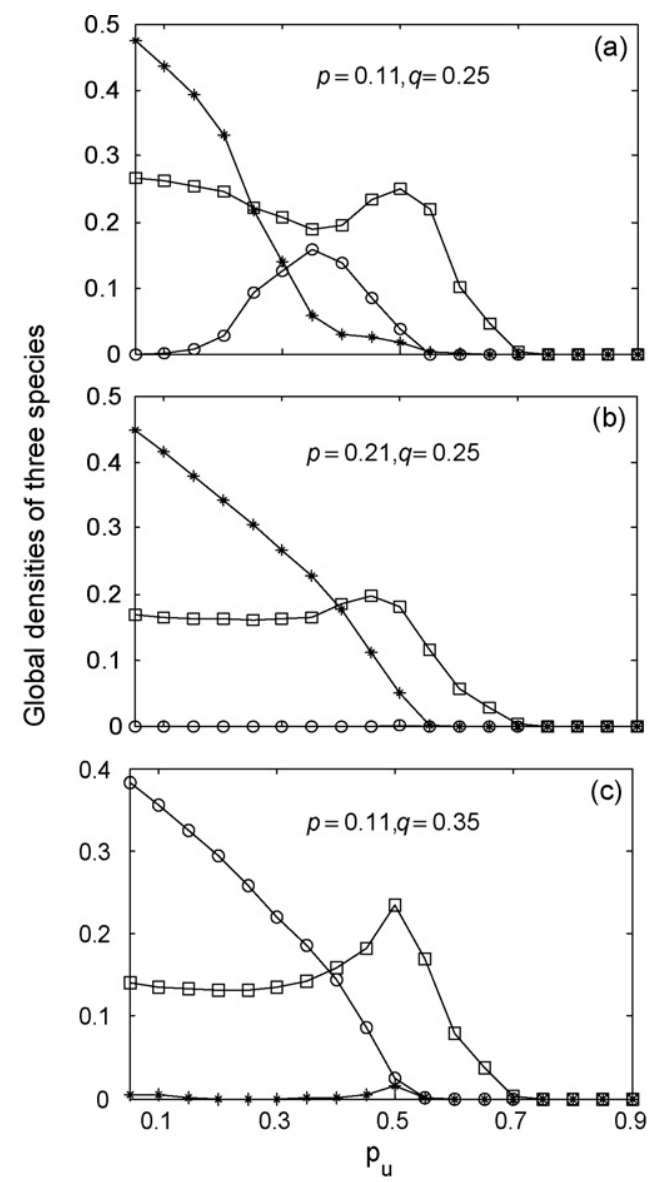

$\square$ Susceptible prey
Predator

Fig. 3 - Three typical patterns of changes in the occupancies (global densities) of three populations (susceptible preys, infected preys and predators) as a function of amount of habitat loss $\left(p_{u}\right)$. The equilibrium global densities of populations derived from eight replicate simulations for each given values of $p_{u}\left(q_{u} / \mathrm{u}=0.6\right)$. Parameter values are: (a) $p=0.11, q=0.25$; (b) $p=0.21$, $q=0.25$; (c) $p=0.11, q=0.35$; Other parameter values are: $r=0.5, w=0.5, d_{1}=0.05, d_{2}=0.11, d_{3}=0.085$.

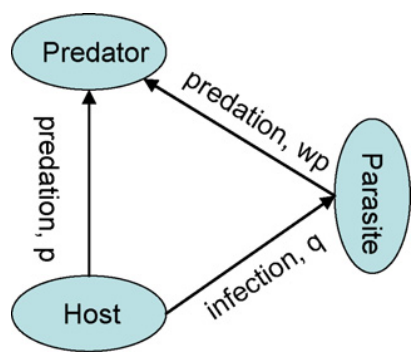

Fig. 4 - Diagram of the eco-epidemiological model that has a same biological mechanism as an intraguild predation system. Here, the infected prey acts as the intraguild prey, predator acts as the intraguild predator, and susceptible prey acts as the shared resource.
Through the above results, we will explore a biologically realistic parameter combination in the following studies, i.e. the parameter combination of Fig. 3a. At high levels of $q_{u} / \mathrm{u}$, the equilibrium of occupancy for the susceptible preys $\left(P_{S}\right)$ declined monotonously with the increase of $p_{u}$, but showed a concave shape when the clustering degree $q_{u}$ is is low (Fig. 5a). When the amount of habitat loss $p_{u}$ was above $0.4, P_{S}$ increased dramatically at first and then decreased gradually with the increase of the clumping degree $q_{\mathrm{u} / \mathrm{u}}$. As for the infected prey, the occupancy $P_{I}$ also showed a complicated pattern with the change of $p_{\mathrm{u}}$ and $q_{\mathrm{u}} \mathrm{u}$ (Fig. 5b). A small amount of habitat loss $\left(p_{u} \leq 0.15\right)$ can make the parasitism of disease successful. But as the amount of habitat loss exceeds $0.15, P_{I}$ declined monotonously with the increase of $p_{u}$, yet increased at first and then decreased monotonously with $q_{u}$ ( (Fig. 5b). While when $p_{\mathrm{u}}$ was very low $\left(p_{\mathrm{u}}<0.15\right), P_{\mathrm{I}}$ declined monotonously with the increase of $q_{u} / \mathrm{u}$ (Fig. 5b). As for the predator populations, the occupancy $P_{\mathrm{P}}$ declined with $p_{\mathrm{u}}$ but increased monotonously with the increase of $q_{u}$ ( (Fig. $5 c$ ). The incidence of the parasitic disease $P_{\mathrm{I}} /\left(\mathrm{P}_{\mathrm{S}}+\mathrm{P}_{\mathrm{I}}\right)$ showed a similar pattern to the infected preys, $P_{I}$ (Fig. $5 d$ ).

The susceptible prey became more aggregated with the increase of $p_{u}$, with an exception at $p_{u}=0.4$ (Fig. 6a). The effect of $p_{\mathrm{u}}$ on $C_{\mathrm{S}}$ was much clearer than the effect of the clustering parameter $q_{u} / u$. The JCS of the infected prey $C_{I}$ increased monotonously with the clustering degree of habitat loss $q_{u} / \mathrm{u}$, and furthermore it showed a valley of low values when the amount of habitat loss $p_{\mathrm{u}}$ was at an intermediate level (Fig. 6b). The JCS of the predators $C_{P}$ increased rapidly with the increase of the amount of lost patches $p_{u}$, but declined with the increase of $q_{u / u}$ (Fig. 6c). Thus, the effect of habitat loss was decisive on the clustering degree of the susceptible prey and predator can be, but was more complicated on the clustering degree of the infected prey.

The extinction threshold of three populations increased monotonously with the clustering degree $q_{u}$ /u (Fig. 7), except for the predator with a minimum threshold at $q_{u / u}=0.25$. It implied that highly clustered habitat loss favors the persistence of populations. Furthermore, for any given clustering degree of habitat loss, the extinction threshold of the susceptible preys was the highest among these three populations. The extinction thresholds for infected prey and for predators were roughly the same, despite the fact that the predator has a higher trophic level than the infected prey (Fig. 7). The extinction threshold curve for the infected prey in Fig. 7 was flatter than the curve for the predator, indicating that the predator was more sensible to the clustering degree of habitat loss than the infected prey. However when facing a high clustering degree of habitat loss $\left(q_{u} / \mathrm{u}>0.75\right)$, the extinction threshold for the infected prey was even lower than for the predator, indicating that the infected prey was more vulnerable than the predator to habitat loss.

\section{Discussion}

In this paper, we have constructed a spatial ecoepidemiological model that incorporated the intensity of habitat loss in terms of the amount $p_{u}$ and its spatial structure $q_{u / u}$. It is the first study that explores the effects of spatial 

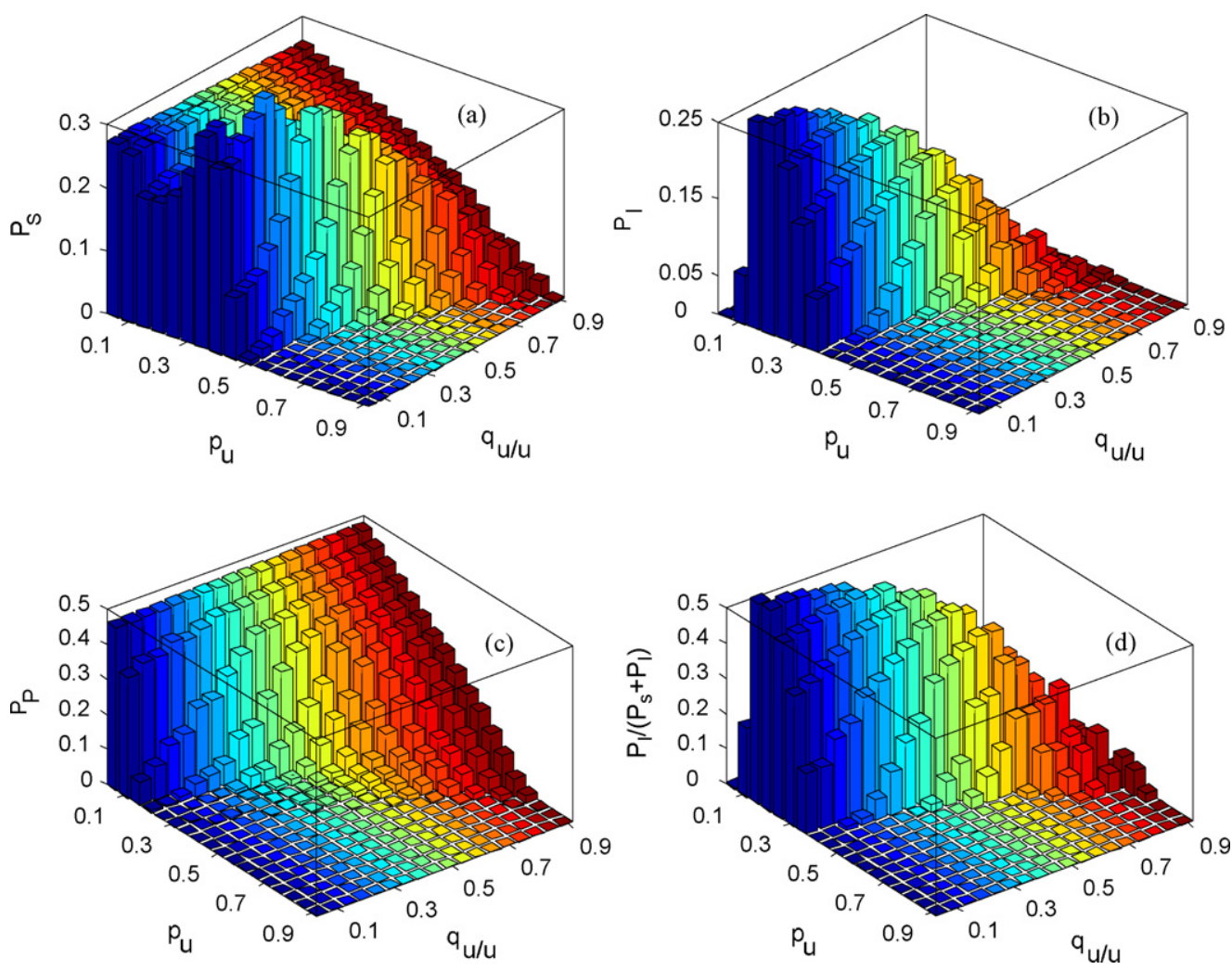

Fig. 5 - (a) The equilibrium of occupancies for the susceptible prey, $P_{S}$, (b) the infected prey, $P_{I}$, and (c) the predator, $P_{P}$, as well as $(d)$ the incidence of disease $P_{I} /\left(P_{S}+P_{I}\right)$, as a function of the amount $\left(p_{u}\right)$ and clustering degree $\left(q_{u} / u\right)$ of habitat loss in the $100 \times 100$ lattice. Parameter values are: $r=0.5, p=0.11, q=0.25, w=0.5, d_{1}=0.05, d_{2}=0.11, d_{3}=0.085$.

habitat loss on such a parasite-host (prey)-predator system. Remarkably, our results confirmed that both the composition and configuration of habitat loss determine the dynamics of the eco-epidemiological system, echoing Hiebeler's (2000, 2004) and Westerberg et al.'s (2005) results on the effect of habitat configuration on the species viability. Meanwhile, the spatial correlation of both the susceptible preys and predators using JCS increased with the increase in habitat loss, which confirms that a highly aggregated distribution of species is a common behavioral strategy when dealing with habitat loss or other environmental stresses (Woodcock et al., 2002; Westerberg et al., 2005). Studies of Diptera communities breeding in fallen fruits, as well as the predator-prey model of some agricultural and phytoplankton systems further support this conclusion (Sevenster and Van Alphen, 1993; Bommarco et al., 2007; El Saadi and Bah, 2007).

The effect of habitat loss on species at different trophic levels is complex, and especially in a so entangled food web (Bascompte and Solé, 1998; Kondoh, 2003; Hiebeler, 2004; Nakagiri and Tainaka, 2004). Some obtained intriguing phenomena (e.g., the concave shape of $P_{S}$ response to habitat loss) also reaffirm this proposition. The concave shape may be due to the following complex interactions: For example, when the amount of lost patches is low, the density of predators declines slightly with the amount of habitat loss, while that of infected preys increases. Hence, the high infection and predation pressures cause the susceptible preys to decrease. At a moderate value of $p_{\mathrm{u}}$, the negative effects of habitat loss causes a sharp decline in the occupancies of infected preys and predators, while the positive effect from the low clustering degree of lost patches is very small, causing the infected preys and predators to decline rapidly. Hence, the susceptible preys show a short-term increase. Another interesting result is that small amounts of habitat loss will benefit the successful invasion of disease, which causes infected preys to increase sharply. This phenomenon is due to the same biological mechanism as in the spatially explicit tri-trophic model (Kondoh, 2003). The reason could arguably be that the predators are always subject to decline when facing habitat loss, and thus the prey is released from low predation pressure.

Furthermore, there exists a clear threshold of clustering degree $\left(q_{u} / u\right)$, at which the infected prey reaches its peak (Fig. 5b). Since a moderately high degree of habitat loss $\left(q_{\mathrm{u} / \mathrm{u}}<0.75\right)$ indicates a higher chance of finding suitable patches beside a suitable patch (Plotnick and Gardner, 1993), the high contact rates will exaggerate the epidemic transmission among prey. However, if the clustering degree of habitat loss is above the 0.75 threshold, suitable patches will start to form large isolated suitable areas in the landscape. On the one hand, once the infected prey becomes extinct in those isolated suitable areas, the disease will become locally extinct; on the other, the high density of predators, which benefits from the very high clustering degree, makes the density of infected preys decrease. Therefore, when the clustering degree of lost patches is too high the disease is also doomed. A similar mechanism could result in a threshold of the incidence 

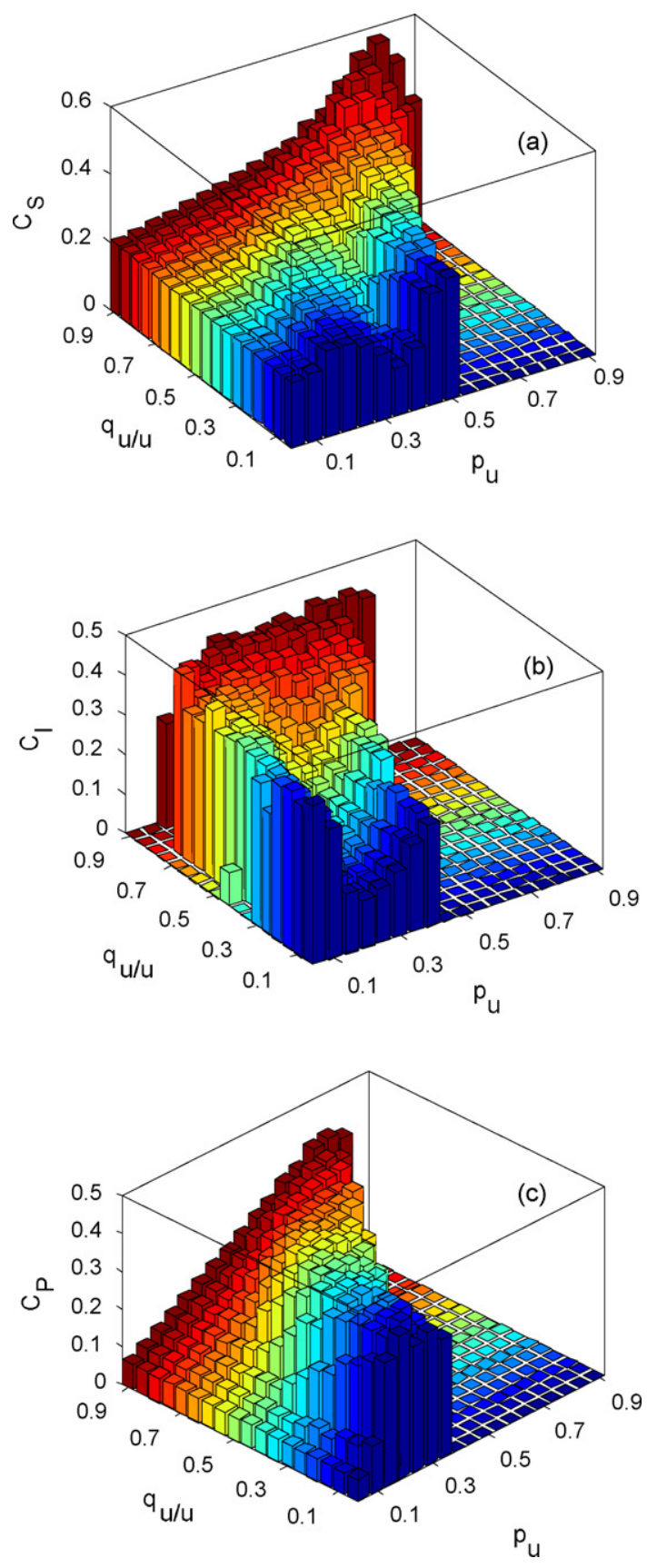

Fig. 6 - (a) The join-count statistics of the susceptible prey, $C_{S}$, (b) the spatial the infected prey, $C_{I}$, and (c) the predator, $C_{P}$, as a function of the amount $\left(p_{u}\right)$ and clustering degree $\left(q_{\mathrm{u} / \mathrm{u}}\right)$ of habitat loss in the $100 \times 100$ lattice. Parameter values are the same as in Fig. 5 .

of disease. These results are consistent with earlier work on a single species model (Hiebeler, 2000).

Finally but very importantly, it is no longer true that the population at the highest trophic level is affected the most by habitat loss. Although unexpected, the existence of such counter-intuitive results is not illogical. When the clustering degree of lost patches is high enough (e.g., $q_{u / u}>0.75$ ), the extinction threshold of the intermediate-trophic species (here the infected prey) is lower than that of the highest-

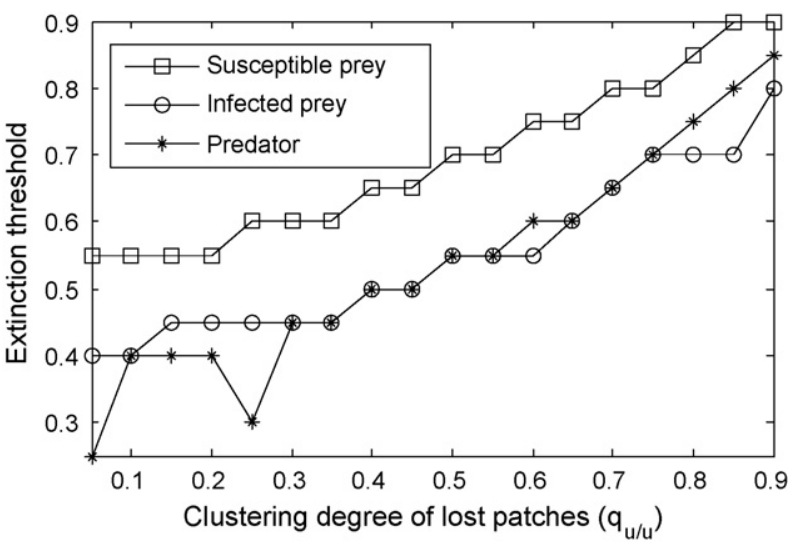

Fig. 7 - The extinction thresholds for three species in the eco-epidemiological model are plotted as a function of the clustering degree of habitat loss $\left(q_{\mathrm{u} / \mathrm{u}}\right)$. Parameter values are the same as in Fig. 5.

trophic species (here the predator). As mentioned above, the parasite-host (prey)-predator system operates according to the same mechanism as an intraguild predation system (Polis and Holt, 1992; Holt and Polis, 1997; Borer, 2006; Su et al., 2008b). Here, in the eco-epidemiological system the infected prey acts as the intraguild prey and the predator acts as the intraguild predator. The infected prey has to balance the conflicting demands of resource acquisition (here the susceptible preys) and predator avoidance (Fig. 4). The predator in the highest trophic has two comestible resources (susceptible and infected preys). As a result, the infected prey becomes extinct before the predator when the lost patches are high clustered.

Biological factors that can alter the dynamics of populations in response to habitat loss are diverse (e.g., Melián and Bascompte, 2002; Lin, 2005; Su et al., 2008a), especially for species embedded in an entangled web with direct and indirect interactions (Fox and Olsen, 2000). Melián and Bascompte (2002) have shown that the order of extinction for two competitors in apparent competition or intraguild predation systems can change significantly depending on the exploration cost of the better competitor. Our result here also emphasizes that both rates of infection and predation, measured as the probabilities per time step, were confirmed to regulate the spatial dynamics of the eco-epidemiological system in response to habitat loss, which is consistent with the results from other epidemic and tri-trophic models (e.g., Kondoh, 2003; Pal et al., 2006). In summary, the species response to habitat loss depends not only on the biological mechanism (such as the structure of food web, attack rates, demographic rates, competition-colonization trade-offs) (Levins, 1969; Tilman, 1994; Melián and Bascompte, 2002; Kondoh, 2003), but also on the external, environmental disturbances (e.g., the spatial structure of habitat loss).

Hiebeler's (2000) algorithm is a prominent method for generating structured landscapes and studying the effect of spatial heterogeneity on population dynamics (e.g., Bjørnstad et al., 1998; Hiebeler, 2000). Other methods for generating heterogeneous landscapes are also available, which are mainly based on the distance function between different types of 
habitat (e.g., Adler and Nuernberger, 1994; Wiegand et al., 1999) and the fractal landscape model (With and King, 2004). The model here supports the reliability of Hiebeler's algorithm and leads to reasonable conclusions in the eco-epidemiological system when taking into account the spatial structure of habitat loss. This study provides important insights by using the spatial design of the landscape and the parasitic disease to conserve/control the endangered/problem species. Some issues are still open. For example, in natural communities, parasites might not only infect host/prey populations but also predators (Chattopadhyay and Bairagi, 2001). A ubiquitous phenomenon in the natural world is that populations always inhabit a spatially structured heterogeneous landscape. Studying the effect of environmental heterogeneity on the broad patterns and structures in ecological communities is definitely worth pursuing. Further studies on the eco-epidemiological systems or other ecological communities should bear in mind the effect of habitat spatial structure on the population dynamics and extinction threshold.

\section{Acknowledgements}

We are grateful to the editor and two anonymous reviewers for their constructive comments and B. Laniewski for logistic help. This work was supported by the National Social Science Foundation of China (04AJL007). C.H. also acknowledges the support from the "Chunhui Scholar" Project of the Ministry of Education of China and the DST-NRF Centre of Excellence for Invasion Biology of South Africa.

\section{REFERE NCES}

Adler, F.R., Nuernberger, B., 1994. Persistence in patchy irregular landscapes. Theor. Popul. Biol. 45, 41-75.

Bairagi, N., Roy, P.K., Chattopadhyay, J., 2007. Role of infection on the stability of a predator-prey system with several response functions-A comparative study. J. Theor. Biol. 248, 10-25.

Bascompte, J., Solé, R.V., 1998. Effects of habitat destruction in a prey-predator metapopulation model. J. Theor. Biol. 195, 383-393.

Bjørnstad, O.N., Andreassen, H.P., Ims, R.A., 1998. Effects of habitat patchiness and connectivity on the spatial ecology of the root vole Microtus oeconomus. J. Anim. Ecol. 67, 127-140.

Bommarco, R., Firle, S.O., Ekbom, B., 2007. Outbreak suppression by predators depends on spatial distribution of prey. Ecol. Model. 201, 163-170.

Borer, E.T., 2006. Does adding biological detail increase coexistence in an intraguild predation model? Ecol. Model. 196, 447-461.

Chattopadhyay, J., Bairagi, N., 2001. Pelicans at risk in Salton Sea-an eco-epidemiological study. Ecol. Model. 136, 103-112.

Deredec, A., Courchamp, F., 2006. Combined impacts of Allee effects and parasitism. Oikos 112, 667-679.

Durrett, R., Levin, S., 1996. Spatial model for the species-area curves. J. Theor. Biol. 179, 119-127.

El Saadi, N., Bah, A., 2007. An individual-based model for studying the aggregation behavior in phytoplankton. Ecol. Model. 204, 193-212.

Fahrig, L., Merriam, G., 1994. Conservation of fragmented populations. Conserv. Biol. 8, 50-59.

Fortin, M.J., Dale, M.R.T., ver Hofe, J., 2002. Spatial analysis in ecology. In: El-Shaarawi, A.H., Piegorsch, W.W. (Eds.),
Encyclopedia of Environmentrics. Wiley, New York, pp. 2051-2058.

Fox, J.W., Olsen, E., 2000. Food web structure and the strength of transient indirect effects. Oikos 90, 219-226.

Garrett, L.J.H., Jones, C.G., Cristinacce, A., Bell, D.J., 2007. Competition or co-existence of reintroduced, critically endangered Mauritius fodies and invasive Madagascar fodies in lowland Mauritius? Biol. Conserv. 140, 19-28.

Hethcote, H.W., Wang, W.D., Han, L.T., Ma, Z.E., 2004. A predator-prey model with infected prey. Theor. Popul. Biol. 66, 259-268.

Hiebeler, D., 2000. Populations on fragmented landscapes with spatially structured heterogeneities: landscape generation and local dispersal. Ecology 81, 1629-1641.

Hiebeler, D., 2004. Competition between near and far dispersers in spatially structured habitats. Theor. Popul. Biol. 66, 205-218.

Hiebeler, D., Morin, B., 2007. The effect of static and dynamic spatially structured disturbances on a locally dispersing population. J. Theor. Biol. 246, 136-144.

Holt, R.D., Polis, G.A., 1997. A theoretical framework for intraguild predation. Am. Nat. 149, 745-764.

Holt, R.D., Lawton, J.H., Polis, G.A., Martinez, N.D., 1999. Trophic rank and the species-area relationship. Ecology 80, 1495-1504.

Hui, C., Li, Z., 2003. Dynamical complexity and metapopulation persistence. Ecol. Model. 164, 201-209.

Hui, C., Li, Z., 2004. Distribution patterns of metapopulation determined by Allee effects. Popul. Ecol. 46, 55-63.

Hui, C., McGeoch, M.A., Warren, M., 2006. A spatially explicit approach to estimating species occupancy and spatial correlation. J. Anim. Ecol. 75, 140-147.

Hui, C., McGeoch, M.A., 2007. Spatial patterns of prisoner's dilemma game in metapopulations. Bull. Math. Biol. 69, 659-676.

Kondoh, M., 2003. Habitat fragmentation resulting in overgrazing by herbivores. J. Theor. Biol. 225, 453-460.

Levins, R., 1969. Some demographic and genetic consequences of environmental heterogeneity for biological control. Bull. Entomol. Soc. Am. 15, 237-240.

Lin, Z., 2005. The ecological order of persisting species during habitat destruction. Ecol. Model. 184, 249-256.

Melián, C.J., Bascompte, J., 2002. Food web structure and habitat loss. Ecol. Lett. 5, 37-46.

Murray, J.D., 1993. Mathematical Biology. Springer Verlag, Berlin.

Nakagiri, N., Tainaka, K., 2004. Indirect effects of habitat destruction in model ecosystems. Ecol. Model. 174, 103-114.

Namba, T., Umemoto, A., Minami, E., 1999. The effects of habitat fragmentation on persistence of source-sink metapopulations in systems with predators and prey or apparent competitors. Theor. Popul. Biol. 56, 123-137.

Noss, R.F., Murphy, D.D., 1995. Endangered species left homeless in Sweet Home. Conserv. Biol. 9, 229-231.

Nowak, M.A., Bonhoeffer, S., May, R.M., 1994. Spatial games and the maintenance of cooperation. Proc. Natl. Acad. Sci. U. S. A. 91, 4877-4881.

Packer, C., Holt, R.D., Hudson, P.J., Lafferty, K.D., Dobson, A.P., 2003. Keeping the herds healthy and alert: implications of predator control for infectious disease. Ecol. Lett. 6, 797-802.

Pal, S., Kundu, K., Chattopadhyay, J., 2006. Role of standard incidence in an eco-epidemiological system: a mathematical study. Ecol. Model. 199, 229-239.

Plotnick, R.E., Gardner, R.H., 1993. Lattices and landscapes. In: Gardner, R.H. (Ed.), Predicting spatial effects in ecological systems. American Mathematical Society, Providence, RI, USA, pp. 129-157.

Polis, G.A., Holt, R.D., 1992. Intraguild predation: the dynamics of complex trophic interactions. Trends Ecol. Evol. 7, 151-154.

Prakash, S., de Roos, A.M., 2002. Habitat destruction in a simple predator-prey patch model: How predators enhance prey persistence and abundance. Theor. Popul. Biol. 62, 231-249. 
Rhodes, C.J., Anderson, R.M., 1997. Epidemic thresholds and Vaccination in a lattice model of disease spread. Theor. Popul. Biol. 52, 101-118.

Satō, K., Iwasa, Y., 2000. Pair approximation for lattice-based ecological models. In: Dieckmann, U., Law, R., Metz, J.A.J. (Eds.), The Geometry of Ecological Interactions: Simplifying Spatial Complexity. Cambridge University Press, Cambridge.

Sayama, H., 2004. Self-protection and diversity in self-replicating cellular automata. Artif. Life 10, 83-98.

Sevenster, J.G., Van Alphen, J.M., 1993. A life history trade-off in Drosophila species and community structure in variable environments. J. Anim. Ecol. 62, 720-736.

Su, M., Hui, C., Zhang, Y., Li, Z., 2008a. Spatiotemporal dynamics of the epidemic transmission in a predator-prey system. Bull. Math. Biol. 70, 2195-2210.

Su, M., Zhang, Y., Hui, C., Li, Z., 2008b. The effect of migration on the spatial structure of intraguild predation in metapopulations. Physica A 397, 4195-4203.

Swihart, R.K., Feng, Z., Slade, N.A., Mason, D.M., Gehring, T.M., 2001. Effects of habitat destruction and resource supplementation in a predator-prey metapopulation model. J. Theor. Biol. 210, 287-303.
Szwabiński, J., Pekalski, A., 2006. Effects of random habitat destruction in a predator-prey model. Physica A 360, 59-70.

Tilman, D., 1994. Competition and biodiversity in spatially structured habitats. Ecology 75, 2-16.

Tilman, D., Kareiva, P., 1997. Spatial ecology: the role of space in population dynamics and interspecific interactions. Princeton University Press, Princeton.

Westerberg, L., Östman, Ö., Wennergren, U., 2005. Movement effects on equilibrium distributions of habitat generalists in heterogeneous landscapes. Ecol. Model. 188, 432-447.

Wiegand, T., Moloney, K.A., Nakes, J., Knauer, F., 1999. Finding the missing link between landscape structure and population dynamics: a spatially explicit perspective. Am. Nat. 154, 605-627.

With, K.A., King, A.W., 2004. The effect of landscape structure on community self-organization and critical biodiversity. Ecol. Model. 179, 349-366.

Woodcock, B.A., Watt, A.D., Leather, S.R., 2002. Aggregation, habitat quality and coexistence: a case study on carrion fly communities in slug cadavers. J. Anim. Ecol. 71, 131-140.

Zhang, F., Hui, C., Han, X.Z., Li, Z.Z., 2005. Evolution of cooperation in patchy habitat under patch decay and isolation. Ecol. Res. 20, 461-469. 